\title{
LES VERRES PHOSPHATES, NOUVEAUX MATÉRIAUX LASER
}

\author{
Par O. K. DEUTSGhbein, G. G. PAUTRAT, I. M. SVIRGHEVSKY, \\ Département «Physique, Chimie, Métallurgie », Centre National d'Études des Télécommunications \\ 92-Issy-les-Moulineaux.
}

\begin{abstract}
Résumé. - Pour cette étude, environ 500 échantillons de verres phosphates dopés au néodyme ont été préparés et on en a étudié les caractéristiques d'absorption et de fluorescence.

Les spectres d'absorption et de fluorescence des verres phosphates sont plus étroits que ceux des verres silicates, borates, germanates et aluminates.

La durée de vie est de l'ordre de $280 \mu \mathrm{s}$.

Des barreaux de verres phosphates ont présenté l'effet laser avec des seuils de 1,02 joule en impulsion et de 720 watts en fonctionnement semi-continu, à la température ambiante : ces valeurs de seuils sont remarquablement plus faibles que celles des autres verres. studied.

Abstract. - About 500 samples of Nd-doped phosphate glasses have been prepared and

Absorption and fluorescence spectra of phosphate glasses are sharper than those of silicate, borate, germanate and aluminate glasses.

The life-time value is about $280 \mu \mathrm{s}$.

Laser rods of phosphate glasses have thresholds of 1.02 joule and of 720 watts in semicontinuous operation at room temperature; these thresholds are markedly lower than those of other glasses.
\end{abstract}

I. Introduction. - Parmi les substances produisant l'effet laser, les matériaux optiques (cristaux et verres) dopés aux terres rares représentent un groupe important, caractérisé par une concentration élevée en atomes actifs; si le volume utile est important, des émissions très énergétiques $(1500 \mathrm{~J})$ et très puissantes $(6 \mathrm{GW})$ sont possibles. Alors qu'il est très difficile de réaliser des monocristaux d'une longueur supérieure à quelques dizaines de $\mathrm{cm}$, cette limitation n'existe pas pour les verres, ceux-ci ayant l'avantage de pouvoir se réaliser en dimensions pratiquement illimitées. Mais, par contre, leur structure est moins bien définie que celle des cristaux et les spectres d'absorption et de fluorescence de l'ion $\mathrm{Nd}^{3+}$ y sont plus flous.

Snitzer a, le premier, obtenu l'effet laser dans un verre silicate dopé au néodyme [1], avec un seuil assez élevé; dès ce moment, il fut vraisemblable que d'autres verres pourraient présenter l'effet laser, avec des seuils de fonctionnement inférieurs; c'est pourquoi nous avons étudié, en détail, les verres phosphates. Ce travail, pour lequel environ 500 échantillons ont été préparés, a été motivé par deux raisons :

I. 1. INTÉRÊts DEs verres Phosphates. - a) Pour éviter des répétitions, puisque la plupart des cher-

Note : Ces travaux furent, à leur début, en partie financés par l'U.S. Department of Army (European Research Office). cheurs (voir la compilation de Snitzer [2], Maurer [3]) ont étudié les verres silicates, borates et boro-silicates; d'autre part, de tels verres dopés au néodyme sont couramment fabriqués industriellement. De plus, les verres phosphates nécessitent des conditions de préparations plus simples que les verres silicates.

b) Au cours de leurs travaux sur la structure des verres, R. Tomaschek et O. Deutschbein [4] trouvèrent, en 1938, que les spectres de fluorescence des terres rares présentent des bandes plus étroites dans les verres phosphates que dans les verres silicates et borates, ce résultat permet donc d'espérer que les verres phosphates auront des seuils lasers plus bas.

I.2. INTÉRÊt DES SUBSTANGES DOPÉES AU NÉODYME. - Depuis les travaux de R. Tomaschek, O. Deutschbein et $H$. Gobrecht [5], on sait que les raies de fluorescence des terres rares correspondent à des transitions entre les niveaux excités et les différents niveaux du multiplet fondamental. Ces derniers sont situés à quelques milliers de $\mathrm{cm}^{-1}$ au-dessus du niveau de base et sont donc, même à la température ambiante, pratiquement inoccupés. On pouvait donc s'attendre à ce que ces matériaux se prêtent bien à la réalisation de lasers à quatre niveaux fonctionnant à la température ambiante. Malgré les travaux de nombreux chercheurs, les transitions vers les niveaux supérieurs du multiplet, pourtant observées en fluorescence, ne donnent pas lieu à un effet laser, bien que ces niveaux soient pratiquement dépeuplés, même à la tempé- 
rature ambiante (cf. Deutschbein et Pautrat [6]). Uniquement dans le cas du néodyme, dont la figure 1 représente les divers niveaux d'énergie pour

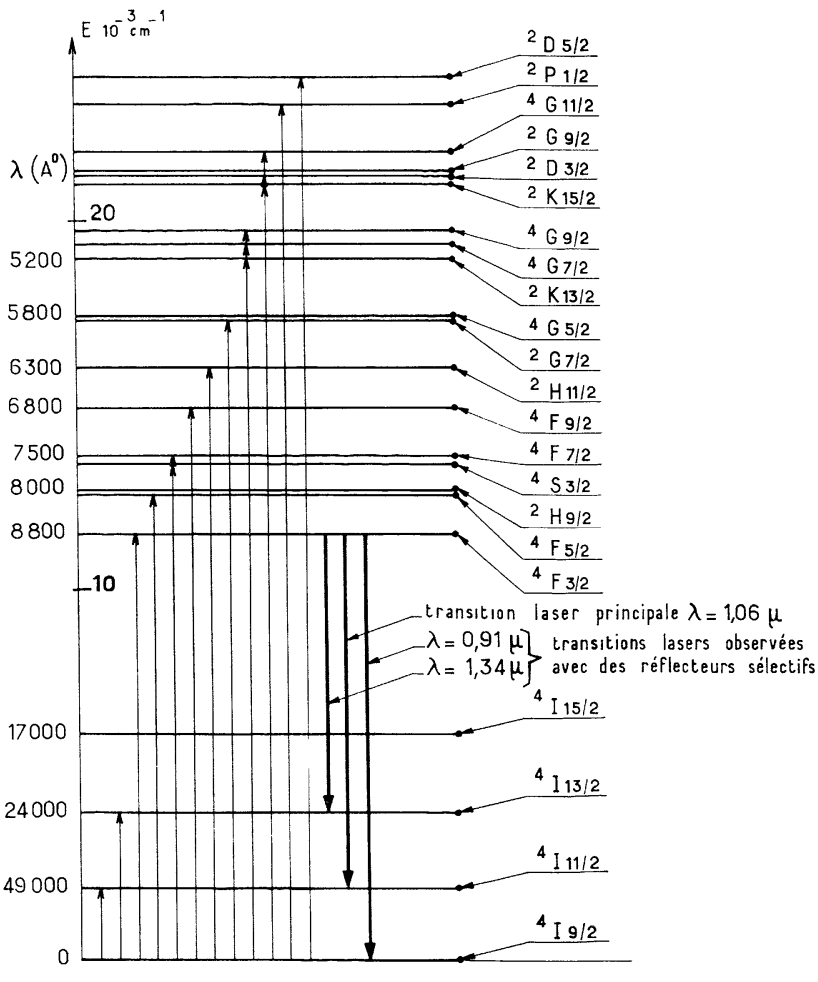

FIg. 1. - Niveaux d'énergie de l'ion $\mathrm{Nd}^{3}+$ isolé.

l'ion trivalent isolé, le niveau final de la transition laser principale, qui est le niveau ${ }^{4} \mathrm{I}_{11 / 2}$, est situé à environ $2000 \mathrm{~cm}^{-1}$ au-dessus du niveau fondamental ${ }^{4} \mathrm{I}_{9 / 2}$. Seules les substances dopées au $\mathrm{Nd}^{+++}$possèdent donc une structure énergétique correspondant à un système à quatre niveaux à la température ambiante. La substance sera particulièrement intéressante si l'évacuation du niveau d'arrivée de la transition laser est suffisamment rapide.

Outre le fait que le Nd permet de réaliser des lasers à quatre niveaux fonctionnant à la température ambiante, il est particulièrement intéressant car son spectre d'absorption est réparti sur le spectre visible et proche infrarouge, ce qui rend le pompage soit par une lampe à incandescence, soit par une lampe à Xénon particulièrement favorable. Enfin, l'émission laser $\mathrm{du} \mathrm{Nd}^{3+}$ a lieu environ à $1,06 \mu$, domaine spectral où l'emploi de détecteurs refroidis n'est pas nécessaire et où l'on dispose de photomultiplicateurs rapides et de convertisseurs d'image à photocathode Cs-Ag.

II. Constitution et préparation des verres phosphates. - II.1. Strugture Des verres Phosphates. - D'après Van Wazer [7], les verres phosphates possèdent des structures différentes suivant leur composi- tion, caractérisée par la valeur du rapport moléculaire :

$$
R=\mathrm{M}_{2} \mathrm{O} / \mathrm{P}_{2} \mathrm{O}_{5}
$$

$M$ étant l'équivalent d'un cation monovalent.

- $\mathrm{O}<R<1$ correspond à la zone d'ultraphosphates ou phosphates à ramifications $\mathrm{M}_{n R} \mathrm{P}_{n} \mathrm{O}_{n \frac{5+R}{2}}$, $n$ étant le nombre d'atomes de phosphore dans l'unité de structure;

- $R=1$, aux métaphosphates ou phosphates cycliques $\left(\mathrm{MPO}_{3}\right)_{n}$;

— et $R>1$, à la zone de polyphosphates ou phosphates linéaires, à chaînes $\mathrm{M}_{n+2} \mathrm{P}_{n} \mathrm{O}_{3 n+1}$ la longueur des chaînes décroît au fur et à mesure que $R$ augmente.

Pour $R=1$, le verre est alors un sel neutre de l'acide phosphorique du type métaphosphate; pour les valeurs de $R<1$, le verre est à caractère acide.

En fait, pour une valeur de $\mathrm{R}$ donnée, on se trouve toujours en présence de mélange de phosphates de différents types, en pourcentages différents suivant l'importance de groupements de base médians, terminaux, à ramifications et même orthophosphates, présents dans la substance. Pratiquement, on classe les phosphates en deux groupes :

a) Les compositions dans lesquelles le rapport $\mathrm{R}$ est inférieur à 1 (ultraphosphates) correspondent à la structure décrite par Zachariasen : réseau tétraédrique dans les interstices duquel se trouvent les ions modificateurs (alcalins, bivalents ou trivalents).

b) Les compositions dans lesquelles le rapport est égal ou supérieur à 1 (méta- et polyphosphates) correspondent à une structure à chaînes avec des cycles possibles en proportion maximum pour des valeurs de $R$ tendant vers 1 (la proportion maximum en cycles est de 7,5\% dans le cas du métaphosphate de sodium et de $11 \%$ dans le cas du métaphosphate de potassium) [7].

La composition joue aussi un rôle sur la facilité de vitrification, et sur les limites de formation du verre qui, de plus, varient d'un cation à un autre : les phosphates de $\mathrm{Li}_{\text {, }} \mathrm{Na}$ donnent des verres pour des rapports $R$ compris entre 0 et 1,5 , les phosphates de $\mathrm{K}, \mathrm{Rb}$, pour des rapports $R$ inférieurs à 1 . Les phosphates de Cs, conformément à la littérature, n'ont pas pu être obtenus sous forme de verre. Les phosphates de $\mathrm{Mg}, \mathrm{Ca}, \mathrm{Sr}$, Cd sont vitreux pour des rapports de $R$ compris entre 0 et 1,2 et le phosphate de zinc jusqu'à 1,7 .

II.2. Éllaboration DES verRes. - Les verres sont formés par refroidissement rapide du produit fondu, mais pour obtenir des verres bien formés il est nécessaire que le mélange soit chauffé à des températures nettement supérieures à celle de la fusion (affinage). Ces températures sont plus ou moins élevées, suivant les cations.

Ges verres sont préparés par réaction des mélanges de carbonates ou d'oxydes métalliques avec l'anhy- 
dride phosphorique et les sels de néodyme. Les mélanges sont préchauffés dans des creusets de platine, à l'air libre, dans un four à moufle, chauffé à $1100^{\circ} \mathrm{C}$, jusqu'à ce que l'on obtienne un mélange fondu clair. Pour l'affinage, le creuset est chauffé dans un four à résistance de rhodium, suivant le cas, à $1400^{\circ} \mathrm{G}$ ou à $1600^{\circ} \mathrm{C}$ pendant 2 ou 3 heures, suivant la composition du mélange. Puis les verres sont coulés dans un moule de graphite et finalement recuits et refroidis lentement, de façon à éliminer les tensions internes d'origine thermique. La capacité de notre équipement de préparation est de l'ordre de $20 \mathrm{~cm}^{3}$. La même méthode est employée pour la préparation des lingots servant à la fabrication des barreaux lasers. Nous utilisons cette méthode, car les dimensions de nos fours ne permettent pas de préparer les mélanges de grands volumes qui sont nécessaires pour obtenir des blocs de bonne homogénéité optique; pour réaliser ceux-ci, il faut des fontes d'au moins 1 litre et un guinandage approprié.

II.3. Composition des différents Verres Étudiés. - Pour étudier l'influence de la composition des verres sur leurs propriétés optiques (cf. § III), nous avons préparé des séries de verres avec diverses proportions de constituants, sans tenir compte de leur stabilité chimique. Nous avons généralement utilisé une concentration en $\mathrm{Nd}$ constante en volume $(0,25$ atomegramme/litre), ce qui correspond à $1,5 \times 10^{20}$ ions de $\mathrm{Nd}^{3+}$ par $\mathrm{cm}^{3}$. Quelques verres d'une concentration variant entre 0,1 et $8 \%$, en poids, ont été préparés afin d'étudier l'influence de la concentration en néodyme sur les propriétés optiques.

Pour cette recherche, environ 500 échantillons de verres ont été préparés; en détail, les séries suivantes de verres ont été étudiées :

a) Verres phosphates simples à cation monovalent, de formule générale : $\mathrm{P}_{2} \mathrm{O}_{5}+\mathrm{R} \cdot \mathrm{X}_{2} \mathrm{O}$, où $\mathrm{X}$ est $\mathrm{Li}, \mathrm{Na}$, $\mathrm{K}, \mathrm{Rb}$.

b) Verres phosphates simples à cation bivalent, de formule générale : $\mathrm{P}_{2} \mathrm{O}_{5}+\mathrm{R}$. YO, où $\mathrm{Y}$ est $\mathrm{Be}, \mathrm{Mg}, \mathrm{Ca}$, $\mathrm{Sr}, \mathrm{Ba}, \mathrm{Zn}$, Cd et $\mathrm{Pb}$.
Pour ces séries, les rapports moléculaires $\mathrm{R}$ ont été variés dans les limites de la phase vitreuse.

c) Verres phosphates à cation trivalent : outre la série $\mathrm{P}_{2} \mathrm{O}_{5}, \mathrm{R} . \mathrm{La}_{2} \mathrm{O}_{3}$, nous avons étudié les séries ternaires : - $\mathrm{P}_{2} \mathrm{O}_{5}, \mathrm{Al}_{2} \mathrm{O}_{3}, \mathrm{X}_{2} \mathrm{O}$ (où $\mathrm{X}$ est $\mathrm{Na}, \mathrm{K}$ et $\mathrm{Rb}$ ); - $\mathrm{P}_{2} \mathrm{O}_{5}, \mathrm{~B}_{2} \mathrm{O}_{3}, \mathrm{Na}_{2} \mathrm{O}$, ces préparations étant difficiles à obtenir à l'état vitreux, sans addition de cation monovalent.

d) Dans le but d'obtenir des verres réunissant les avantages des verres à cation alcalin (ceux-ci ont une bande de fluorescence étroite mais une médiocre stabilité chimique) et ceux des verres à cation bivalent (excellente stabilité chimique); nous avons préparé des verres mixtes, mélanges de verres à rapport moléculaire unité :

$$
\frac{x}{2}\left(\mathrm{P}_{2} \mathrm{O}_{5}+\mathrm{X}_{2} \mathrm{O}\right)+\left(\mathrm{P}_{2} \mathrm{O}_{5}+\mathrm{YO}\right)
$$

dont la formule globale est :

$$
\left(\mathrm{PO}_{3}\right)_{x+2} \mathrm{X}_{x} \mathrm{Y}
$$

- où $\mathrm{X}$ est un métal alcalin ( $\mathrm{Li}, \mathrm{Na}, \mathrm{K}, \mathrm{Rb}$ et $\mathrm{Cs})$, - et où $\mathrm{Y}$ est un métal bivalent $(\mathrm{Be}, \mathrm{Mg}, \mathrm{Ca}, \mathrm{Sr}$, $\mathrm{Ba}, \mathrm{Zn}$ et $\mathrm{Cd}$ ),

et ce pour $x$ égal à $0,5,1,2,3,4$ et 5 .

e) A titre de comparaison, des verres phosphates dont les compositions, décrites dans des brevets [7, 8, 9], comportent un grand nombre de composants, ont aussi été préparés.

\section{RESULTATS DE MESURES}

III. 1. Spectres d'absorption. - Un spectrophotomètre à double faisceau Cary $14 \mathrm{R}$ a servi à enregistrer et mesurer, avec une résolution de l'ordre de $1 \AA$, les bandes d'absorption de l'ion néodyme, dans les différents verres, entre 0,3 et $0,9 \mu$.

Les bandes d'absorption des verres phosphates sont remarquablement plus étroites que celles des verres silicates et, contrairement à ceux-ci, les bandes à 0,58 , 0,74 et $0,80 \mu$ y ont des intensités comparables entre elles.

La figure 2 donne tout le spectre d'absorption

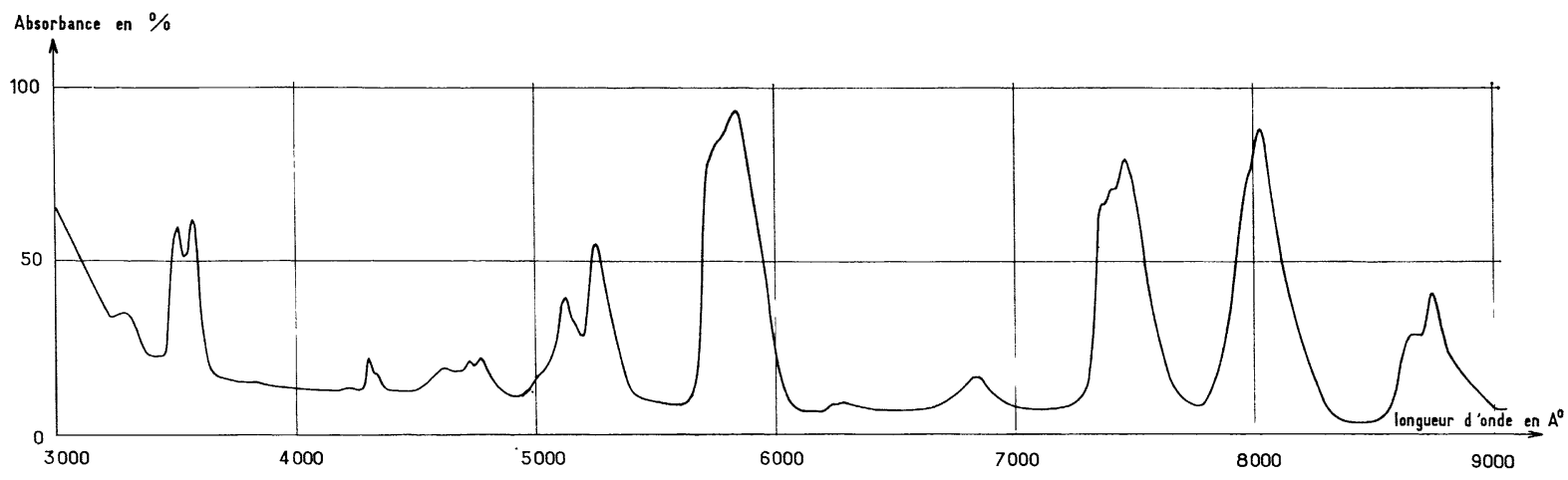

FIG. 2. - Variation de l'absorbance d'un verre phosphate (avec $\mathrm{SrO} / \mathrm{P}_{2} \mathrm{O}_{5}=0,8$ ), épaisseur 4,5 $\mathrm{mm}$ en fonction de la longueur d'onde. 
entre 0,3 et $0,9 \mu$ d'un verre phosphate bivalent au strontium $\left(\mathrm{SrO} / \mathrm{P}_{2} \mathrm{O}_{5}=0,8\right)$ dopé avec $2 \%$ en poids de $\mathrm{NdF}_{3}$, on y remarque les bandes d'absorption caractéristiques de l'ion $\mathrm{Nd}^{3+}$ à $0,35,0,52,0,58,0,68$, $0,74,0,80$ et $0,87 \mu$.

III.2. Spectres de fluorescence. - Le but de ces mesures est de connaître la position spectrale de l'intensité maximum de fluorescence, ainsi que la largeur de la bande de fluorescence.

III.2.1. Position SPegtrale du maximum De fluoRESGence. - La longueur d'onde $\lambda_{0}$, où se situe le maximum d'intensité de fluorescence, varie peu dans une même classe de verre et est surtout sensible au rapport moléculaire $R: \lambda_{0}$ augmente avec $R$ et croît, par exemple, de $10515 \AA$ à $10540 \AA$ lorsque $R$ varie de 0,6 à 1,2 dans un verre $\mathrm{P}_{2} \mathrm{O}_{5}+\mathrm{R}$. CdO. Pour les verres pour lesquels $R=1$, et en particulier les verres mixtes,

$$
\lambda_{0}=10532 \pm 5 \AA
$$

III.2.2. LARGEUR DE BANDE DE FLUORESGENGE. La largeur de la raie de fluorescence (mesurée à mihauteur) est un paramètre important dans la théorie de l'effet laser. Le coefficient d'absorption $\alpha$ (ou d'amplification en cas d'inversion de population) est relié aux caractéristiques du matériau par diverses formules, suivant que la raie envisagée a un profil de Gauss [10], cas des gaz ou un profil de Lorentz [11]. Ces formules indiquent que l'absorption (ou l'amplification) est d'autant plus grande que la largeur de raie est plus faible, à force d'oscillateur égale.

La largeur, mesurée à mi-hauteur, du spectre de fluorescence d'un verre dopé n'est pas du tout celle qui intervient dans les formules citées ci-dessus où l'on a admis que l'émission avait lieu sur une raie unique et simple comme c'est le cas dans les gaz. La situation indiquée par la figure 1 n'est valable que pour l'ion $\mathrm{Nd}$ isolé et déjà, dans les cristaux, les divers niveaux mis en jeu sont décomposés en plusieurs composantes par le champ cristallin qui lève ainsi la dégénérescence de ces niveaux. Le spectre de fluorescence de l'ion devient alors complexe, formé de plusieurs raies se chevauchant plus ou moins les unes les autres. Ainsi, dans le $\mathrm{CaWO}_{4}$, dopé au néodyme, le niveau de départ ${ }^{4} F_{3 / 2}$ de la transition laser est dédoublé et le niveau final ${ }^{4} I_{11 / 2}$ est décomposé en 5 sous-niveaux [12], ce qui donne lieu à 10 transitions de fluorescence qui participent toutes à la perte d'énergie par émission spontanée. Dans le cas de verres dopés, les écarts entre les niveaux sont variables d'un environnement à un autre, et il en résulte que le spectre de fluorescence, qui est alors la superpusition des contributions de chacun de ces environnements, est plus flou que dans les cristaux. Dans le cas de telles bandes de fluorescence complexes, la largeur mesurée à mi-hauteur peut conduire à des conclusions erronées : ainsi la raie représentée figure $3 a$ a une largeur surestimée, tandis


FIG. 3. - Influence de la complexité d'une raie de fluorescence sur la largeur mesurée à mi-hauteur.

que la raie de la figure $3 b$ donne lieu à une valeur trop faible. Ce dernier cas est réalisé dans l'exemple de la figure $4 b$.

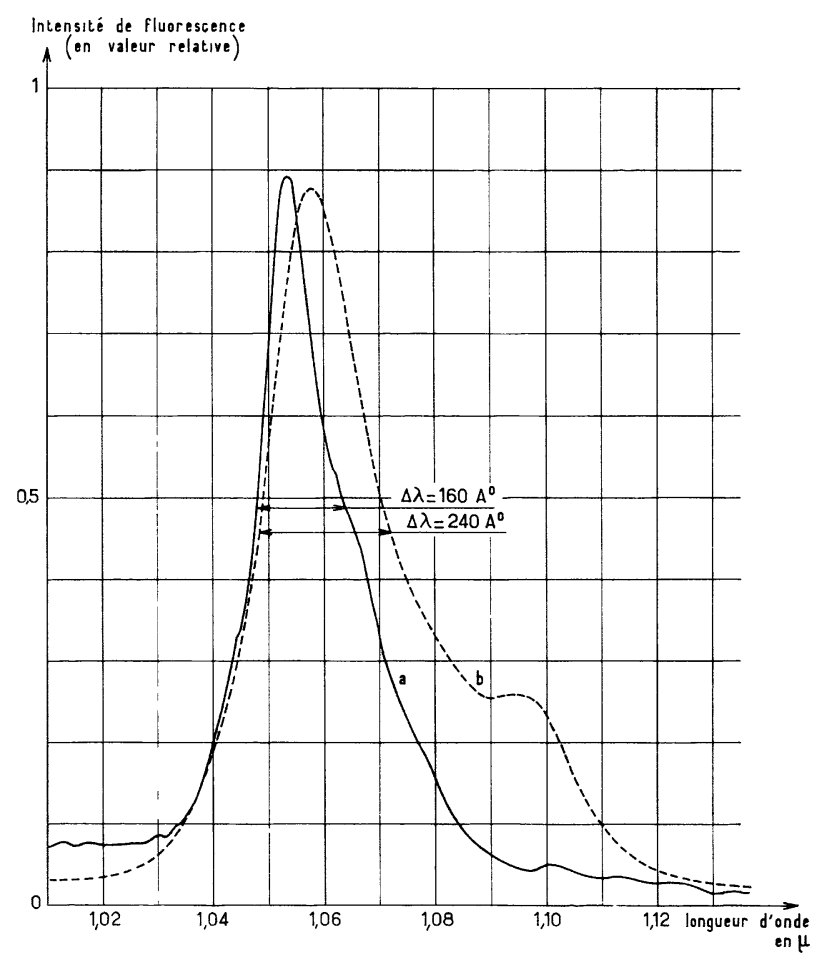

FIG. 4. - Spectres de fluorescence, autour de 1,06 $\mu$ :

a) d'un verre phosphate alcalin-zinc.

b) d'un verre laser silicate commercial.

Pour tenir compte de la dispersion de l'énergie d'excitation dans tout le spectre de fluorescence par émission spontanée, par rapport à l'énergie laser émise à la longueur d'onde qui correspond à la transition la plus intense, nous avons introduit la notion de « largeur de raie effective » pour caracté- 
riser les spectres d'émission des diverses substances : cette « largeur de raie effective » $\delta \lambda$ est définie par où

$$
\delta \lambda=H A_{\mathrm{t}} / I_{\max }
$$

- $A_{\mathrm{t}}$ est l'aire totale de la bande de fluorescence enregistrée avec ses diverses composantes.

- $I_{\max }$ est l'intensité de la bande de fluorescence en son maximum.

- $H$ est un coefficient, tel que, pour une raie type simple, $\delta \lambda$ coïncide avec sa largeur réelle $\Delta \lambda$ à mihauteur; suivant que la raie type choisie a un profil triangulaire, de Gauss ou de Lorentz, $H$ égale 1 ou 0,94 ou $2 / \pi$. Le spectre d'émission des solides fluorescents est en général composé de bandes au profil de Lorentz.

Ainsi les deux spectres représentés figure 3 , bien qu'ayant des $\Delta \lambda$, mesurées à mi-hauteur, très différentes, ont des largeurs effectives égales.

Bien entendu, cette largeur $\delta \lambda$ n'est pas la largeur naturelle de la transition sur laquelle s'effectue l'émission laser.

Les spectres de fluorescence émis autour de 1,06 $\mu$ par les verres furent enregistrés avec un spectrophotomètre Cary 14 légèrement modifié, l'excitation étant effectuée avec la lumière d'une lampe à incandescence convenablement filtrée. Sur ces enregistrements nous avons mesuré la largeur à mi-hauteur $\Delta \lambda$ de la bande de fluorescence et déterminé ensuite, par intégration graphique, $\delta \lambda$.

III .2.2.1. Influence des conditions de préparation. - La durée et la température de l'affinage n'ont qu'une faible influence sur la largeur de bande, lorsque cette température est supérieure à celle de formation du verre. Une température de $1300^{\circ} \mathrm{C}$ est généralement suffisante, sauf pour les verres au $\mathrm{Be}$, au $\mathrm{Mg}$ et pour les verres à cation trivalent.

III.2.2.2. Influence de la concentration en néodyme. La concentration en $\mathrm{Nd}$ a très peu d'influence sur la largeur de la raie de fluorescence, celle-ci s'élargissant pour des concentrations en néodyme supérieures à $2 \%$ en poids.

III.2.2.3. Influence de la composition. - La largeur de la bande de fluorescence des verres phosphates à cation alcalin est inférieure à celle des verres à cation bivalent, et dans tous les cas elle diminue lorsque le poids atomique du cation augmente, ainsi qu'on le voit dans le tableau ci-dessous, relatif à des verres $\mathrm{P}_{2} \mathrm{O}_{5}+0,6 \mathrm{X}_{2} \mathrm{O}$ et $\mathrm{P}_{2} \mathrm{O}_{5}+\mathrm{YO}$ :

\begin{tabular}{cccccccccccc} 
GAtion & $\mathrm{Li}$ & $\mathrm{Na}$ & $\mathrm{K}$ & $\mathrm{Rb}$ & $\mathrm{Be}$ & $\mathrm{Mg}$ & $\mathrm{Ca}$ & $\mathrm{Zn}$ & $\mathrm{Sr}$ & $\mathrm{Cd}$ & $\mathrm{Ba}$ \\
- & - & - & - & - & - & - & - & - & - & - & - \\
$\Delta \lambda($ en $\AA$ ) & 206 & 182 & 185 & 179 & 302 & 262 & 250 & 249 & 234 & 232 & 226 \\
$\delta \lambda($ en $\AA$ ) & 147 & 135 & 139 & 135 & 226 & 212 & 196 & 192 & 176 & 176 & 170 \\
\hline
\end{tabular}

Il est remarquable que les verres mixtes (cf. II $.3 d$ ) contenant à la fois des cations monovalents et des cations bivalents ont des bandes de fluorescence encore plus étroites. $\Delta \lambda$ et $\delta \lambda$ diminuent :

- lorsque les poids atomiques des cations monovalent ou bivalent augmentent;

- lorsque, à constituants donnés, $x$ augmente, c'est-à-dire lorsque la proportion d'alcalin est accrue.

Le tableau ci-après indique quelques exemples des résultats obtenus :

\begin{tabular}{|c|c|c|}
\hline Composition & $\Delta \lambda($ en $\AA)$ & $\delta \lambda($ en $\AA)$ \\
\hline$\left(\mathrm{PO}_{3}\right)_{6} \mathrm{ZnLi}_{4}$ & 204 & 160 \\
\hline$\left(\mathrm{PO}_{3}\right)_{6} \mathrm{ZnK}_{4}$ & 171 & 135 \\
\hline$\left(\mathrm{PO}_{3}\right)_{6} \mathrm{CdK}_{4}$ & 166 & 132 \\
\hline$\left(\mathrm{PO}_{3}\right)_{6} \mathrm{CdCs}_{4}$ & 160 & 132 \\
\hline$\left(\mathrm{PO}_{3}\right)_{2,5} \mathrm{BaK}_{0,5}$ & 206 & 157 \\
\hline$\left(\mathrm{PO}_{3}\right)_{3} \mathrm{BaK}$ & 200 & 152 \\
\hline$\left(\mathrm{PO}_{3}\right)_{4} \mathrm{BaK}_{2}$ & 182 & 134 \\
\hline$\left(\mathrm{PO}_{3}\right)_{6} \mathrm{BaK}_{4}$ & 153 & 132,5 \\
\hline
\end{tabular}

Les verres simples à cation trivalent ont des spectres larges; ainsi, pour le verre au lanthane, de rapport moléculaire unité, $\Delta \lambda$ et $\delta \lambda$ ont pour valeurs 257 et
$200 \AA$ respectivement. De façon générale, même ajoutés en faible proportion, les cations trivalents élargissent le spectre de fluorescence : voici, par exemple, pour un verre au sodium (tel que $\mathrm{Na}_{2} \mathrm{O} / \mathrm{P}_{2} \mathrm{O}_{5}=1 / 3$ ), l'effet de l'addition de $\mathrm{Al}_{2} \mathrm{O}_{3}$ (en \% en poids) :

$$
\begin{array}{cccccc}
\% \mathrm{Al}_{2} \mathrm{O}_{3} & 0 & 2,5 & 5 & 10 & 15 \\
-\Delta \lambda & - & - & - & - & - \\
182 \AA & 193 \AA & 197 \AA & 206 \AA & 236 \AA
\end{array}
$$

Cet élargissement est, à concentration de $\mathrm{Al}_{2} \mathrm{O}_{3}$ constante (égale à $15 \%$ ci-dessous), encore plus important lorsque le rapport $\mathrm{R}=\mathrm{Na}_{2} \mathrm{O} / \mathrm{P}_{2} \mathrm{O}_{5}$ augmente :

$$
\begin{array}{cccc}
\frac{R}{-} & 0,33 & 0,5 & 1 \\
\Delta \lambda(\text { en } \AA) & 236 & 242 & 248
\end{array}
$$

On constate que pour les verres phosphates le rapport $\Delta \lambda / \delta \lambda$ est généralement voisin de 1,3 , ceci étant dû à la structure complexe particulière de la bande de fluorescence de l'ion néodyme dans les verres phosphates.

Les verres cités dans certains brevets ( $\S$ II.3 $e$ ) possèdent des bandes de fluorescence dont la largeur (270-300 §) est semblable à celle des verres silicates. 
Ce résultat est d'ailleurs général : la présence de nombreux constituants provoque l'élargissement de la bande de fluorescence.

III .2.2.4. Comparaison avec d'autres types de verres. Le tableau ci-après donne une comparaison des valeurs de $\Delta \lambda$ et $\delta \lambda$ (en $\AA$ ) pour quelques types de verres :

\begin{tabular}{lll} 
& $\Delta \lambda$ & \multicolumn{1}{c}{$\delta \lambda$} \\
& - & - \\
Verre $\left(\mathrm{PO}_{\mathbf{3}}\right)_{\mathbf{6}} \mathrm{BaK}_{\mathbf{4}}$ & 153 & 132,5 \\
Verre silicate $\mathrm{Ba}, \mathrm{K}$ & 230 & 210 \\
Verre germanate & 310 & 225 \\
Verre aluminate & 408 & 295
\end{tabular}

Les verres phosphates ont donc des spectres de fluorescence nettement plus étroits que les autres verres; ceci apparaît également sur la figure 4, qui est l'enregistrement des bandes de fluorescence d'un verre phosphate alcalin-zinc et d'un verre silicate commercial. On remarque que la longueur d'onde correspondant au maximum d'intensité est également différente pour chacun des verres.

III. 3. Mesures de la durée de vie du niveau métastable. - La durée du niveau d'énergie métastable ${ }^{4} \mathrm{~F}_{3 / 2}$, qui est le niveau initial de la transition laser, a été mesurée par la décroissance de la fluorescence, pour tous les verres préparés, en utilisant la méthode décrite par O. Deutschbein et F. Auzel [13]. Cette méthode a une précision de $10 \%$.

Les résultats sont les suivants :

III.3.1. INFLUENGE DES GONDITIONS DE PRÉPARATION. - La durée de vie ne prend une valeur maximum que

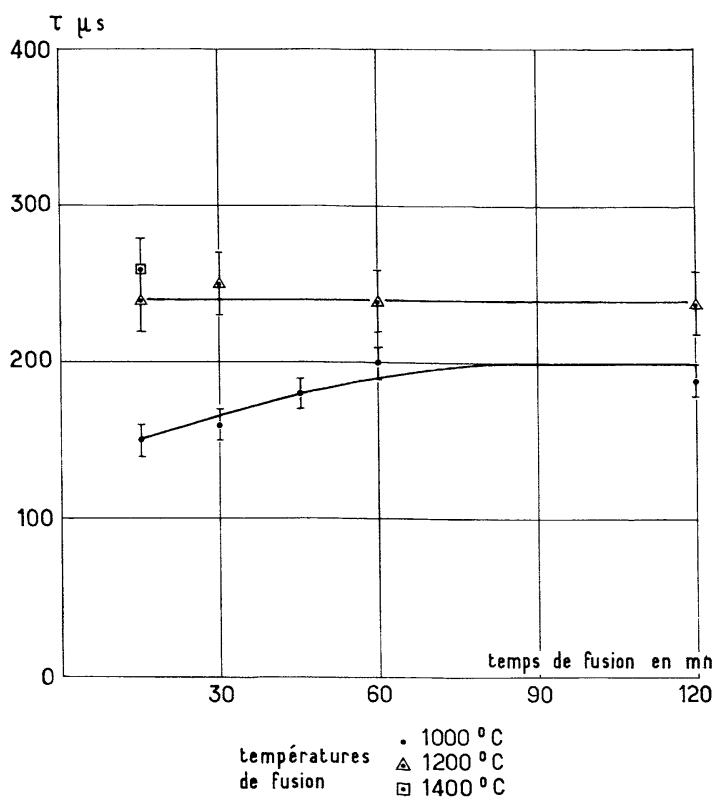

FIG. 5. - Variation de la durée de vie du niveau ${ }^{4} \mathrm{~F}_{3 / 2}$ en fonction du temps de fusion pour différentes températures de fusion, pour un verre alcalin à l'oxyde de zinc. lorsqu'une température de formation assez élevée est atteinte et ce, avec un temps de fusion suffisant. Ainsi sur la figure 5 sont représentés à la fois les effets de la température de fusion et de la durée de fusion sur un verre phosphate alcalin à l'oxyde de zinc, dopé avec $\mathrm{NdF}_{3}$, la durée de vie atteignant une valeur maximum pour des températures et des durées supérieures à $1200^{\circ} \mathrm{C}$ et 30 minutes respectivement.

III.3.2. INFLUENGE DE LA GONGENTRATION DE NÉODYME. - L'influence de la concentration en $\mathrm{Nd}$ est à peu près la"même pour tous les verres phosphates : la durée

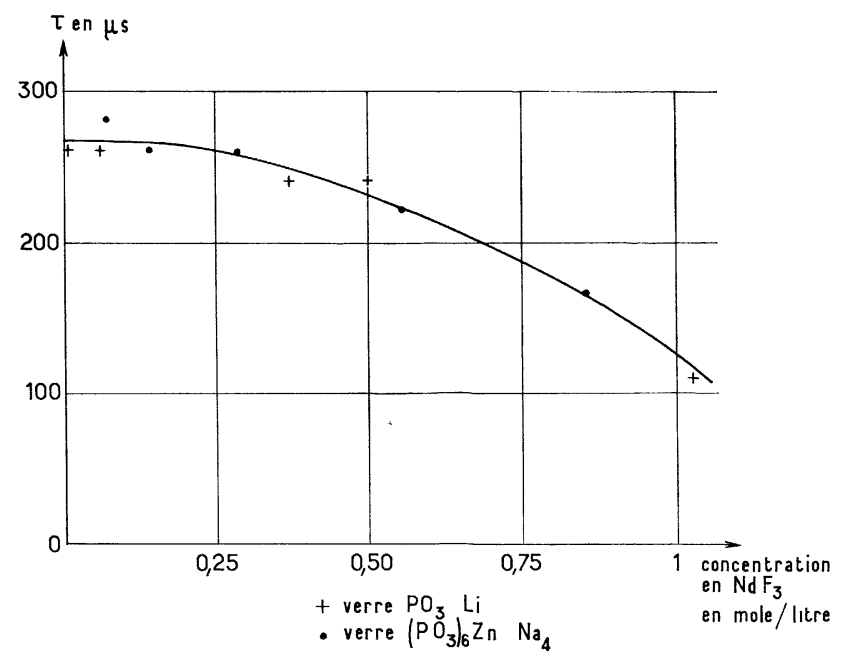

FIG. 6. - Variation de la durée de vie du niveau ${ }^{4} \mathrm{~F}_{3 / 2}$ dans des verres phosphates en fonction de la concentration en $\mathrm{NdF}_{3}$.

de vie reste constante à faible concentration puis décroît, elle prend une valeur moitié pour une concentration en $\mathrm{Nd}$ égale à peu près à un Atg/litre. La figure 6 représente ces variations pour deux types de verres phosphates.

III . 3. 3. INFLUENGE DE LA GOMPOSITION DU VERRE. Les verres phosphates ont généralement des durées de vie situées entre 200 et $300 \mu$ s dans la mesure où ils sont vitreux. D'autre part, les échantillons dévitrifiés ont des durées de vie très courtes : de l'ordre de $60 \mu \mathrm{s}$.

Pour quelques verres alumino-phosphates, les durées de vie sont de 300 à $380 \mu \mathrm{s}$. Ces valeurs s'approchent de celles des verres silicates qui sont de l'ordre de $500 \mu \mathrm{s}$.

Les verres germanates et aluminates possèdent des durées de vie comprises entre 200 et $250 \mu \mathrm{s}$.

III.4. Calcul des forces d'oscillateur. - La force d'oscillateur caractérise la probabilité de transition entre deux niveaux et sa connaissance permet de calculer la concentration en $\mathrm{Nd}$ optimum pour une géométrie de barreau laser donnée. 
A partir des enregistrements des spectres d'absorption, par intégration graphique, nous avons calculé les forces d'oscillateur des différentes transitions (voir § III.1) d'après la formule :

$$
f=\frac{m c^{2}}{\pi e^{2} N} \int K\left(\nu^{*}\right) \mathrm{d} \nu^{*}
$$

où : $K\left(\nu^{*}\right)=$ coefficient d'absorption,

$\nu^{*}=$ nombre d'onde $=1 / \lambda$,

$m, e=$ masse et charge de l'électron,

$N=$ nombre d'ions par $\mathrm{cm}^{3}$,

$c \quad=$ vitesse de la lumière.
Comme on le voit sur la figure 2, la force d'oscillateur est différente pour chaque bande d'absorption.

III.4.1. InFLuenGe DE LA COMPOSITION. - Le rapport moléculaire entre $\mathrm{P}_{2} \mathrm{O}_{5}$ et les divers oxydes stabilisateurs a une influence réelle sur les forces d'oscillateur des diverses bandes d'absorption : celles-ci augmentent avec la proportion en $\mathrm{P}_{2} \mathrm{O}_{5}$ (i.e. quand $\mathrm{R}$ devient $<1$ ).

La nature du cation a peu d'influence : à titre de comparaison, on trouve, ci-dessous, les résultats relatifs aux verres $\mathrm{P}_{2} \mathrm{O}_{5}+\mathrm{Na}_{2} \mathrm{O}, \mathrm{P}_{2} \mathrm{O}_{5}+\mathrm{BaO},\left(\mathrm{PO}_{3}\right)_{6} \mathrm{BaK}_{4}$ et à un verre silicate commercial; ces valeurs sont multipliées par $10^{6}$ :

\begin{tabular}{|c|c|c|}
\hline & $\lambda=\underline{0,52 \mu}$ & $\lambda=\underline{0,58 \mu}$ \\
\hline $\mathrm{P}_{2} \mathrm{O}_{5}+\mathrm{Na}_{2} \mathrm{O}$ & 6,05 & 15,8 \\
\hline $\mathrm{P}_{2} \mathrm{O}_{5}+\mathrm{BaO}$ & 5,6 & 15,9 \\
\hline$\left(\mathrm{PO}_{3}^{\circ}\right)_{6} \mathrm{BaK}_{4}$ & 5,8 & 16,1 \\
\hline Verre silicate $\mathrm{Ba}, \mathrm{K}$ & 10,8 & 33,3 \\
\hline
\end{tabular}

Avec les verres silicates, la plus grande différence réside dans les bandes à 0,51 et $0,58 \mu$ qui ont des forces d'oscillateur deux fois plus grandes dans les verres silicates que dans les verres phosphates. Dans ces derniers, la plus grande égalité des forces d'oscillateur est un caractère très favorable pour l'excitation des barreaux lasers, en assurant une homogénéité dans l'absorption de la lumière de pompage.

III.4.2. INFLUENGE DE LA GONGENTRATION EN NÉODYME. - La somme des forces d'oscillateur est pratiquement indépendante de la concentration en dopant.

III.4.3. INFLUENGE DES GONDITIONS DE PRÉPARATION. - Si l'on élève la température d'affinage du verre, les forces d'oscillateur de chacune des bandes d'absorption augmentent légèrement et ce, surtout dans le spectre visible.

III.5. Mesures de différents paramètres physiques. - Pour tous les verres préparés, nous avons aussi mesuré : l'indice de réfraction; la densité; la stabilité chimique.

III.5.1. INDIGe DE RÉfraction ET DENSITÉ. L'indice de réfraction a été mesuré avec un réfractomètre d'Abbe, pour la raie $D$ du sodium.

La densité des échantillons a été mesurée par immersion dans un liquide où le verre n'est pas soluble : le tétrachlorure de carbone.

La densité et l'indice de réfraction, pour des verres de même composition, augmentent avec la teneur en $\mathrm{Nd}$ : ces mêmes paramètres augmentent avec le rapport $R$, pour tous les cations.

- La figure 7 est relative à quelques verres phosphates bivalents avec $R=\mathrm{YO} / \mathrm{P}_{2} \mathrm{O}_{5}=1$ pour $\mathrm{Y}=\mathrm{Be}, \mathrm{Mg}, \mathrm{Ca}, \mathrm{Zn}, \mathrm{Sr}$, Cd et Ba.

$\begin{array}{cccc}\lambda=0,68 \mu & \lambda=0,74 \mu & \lambda=0,80 \mu & \lambda=0,88 \mu \\ - & - & - & - \\ 0,27 & 6,7 & 7 & 2 \\ 0,5 & 6,6 & 6,7 & 1,74 \\ 0,46 & 7,1 & 7,7 & 2,1 \\ 0,36 & 8,1 & 7,7 & 1,88\end{array}$

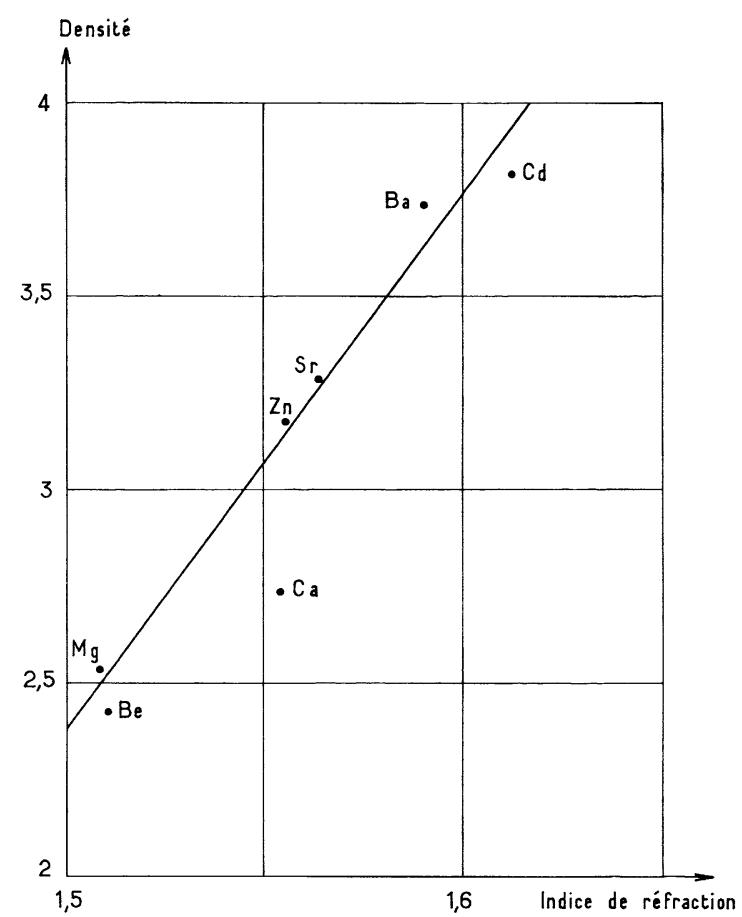

Fig. 7. - Densité et indice de réfraction de -verres phosphates bivalents d'un rapport moléculaire égal à 1 .

\section{Facteurs de densité.}

La densité d'un verre est reliée à ses constituants par une relation additive, qui s'exprime en assignant à chaque oxyde constituant un facteur, grâce auquel la densité peut être calculée, approximativement, au moyen d'une formule du type :

$$
\frac{100}{D}=\frac{x^{\prime}}{d^{\prime}}+\frac{x^{\prime \prime}}{d^{\prime \prime}}+\ldots \frac{x^{n}}{d^{n}}
$$


où $D$ est la densité du verre; $x^{\prime}, x^{\prime \prime}, \ldots x^{n}$ sont les pourcentages en poids des constituants et $d^{\prime}, d^{\prime \prime}$, $d^{\prime \prime \prime}, \ldots d^{n}$ sont les facteurs correspondants [14]. A l'aide de cette formule, nous avons calculé les facteurs de densité des divers oxydes dans les verres phosphates et également celui du $\mathrm{P}_{2} \mathrm{O}_{5}$.

Dans le tableau ci-dessous sont indiquées ces valeurs ainsi que les densités des mêmes oxydes à l'état cristallin :



Les facteurs de densité sont généralement plus grands que la densité de l'oxyde pur et ce, surtout pour les oxydes donnant une bande de fluorescence étroite.

Le facteur de densité de $\mathrm{P}_{2} \mathrm{O}_{5}$ varie avec les divers constituants; ainsi, pour des verres simples, les résultats sont les suivants :

avec $\mathrm{La}_{2} \mathrm{O}_{3}$ le facteur de densité de $\mathrm{P}_{2} \mathrm{O}_{5}$ est 2,27

- $\mathrm{CaO} \quad-\quad$ - $\quad 2,37$

$\begin{array}{llll}-\mathrm{BeO} & - & - & 2,39\end{array}$

$\begin{array}{llll}-\mathrm{SrO} & - & - & 2,39\end{array}$

- $\mathrm{ZnO} \quad-\quad-\quad-\quad 2,45$

- $\begin{array}{llll}\mathrm{BaO} & - & - & 2,57\end{array}$

- $\begin{array}{lllll}\mathrm{CdO} & - & - & 2,58\end{array}$

Il est remarquable que les cations donnant les spectres de fluorescence les plus étroits sont ceux où le $\mathrm{P}_{2} \mathrm{O}_{5}$ a un facteur de densité le plus grand.

III .5 .2. DÉTERMINATION DE L'INDIGE DE SOLUBILITÉ. - La stabilité chimique des verres a été caractérisée par l'indice de solubilité $S$, déterminé suivant une méthode classique [15].

Les résultats sont les suivants :

- les verres phosphates alcalins sont complètement solubles dans les conditions de l'essai;

- les verres phosphates alcalino-terreux sont plus résistants, spécialement le verre phosphate au Be qui est presque insoluble $(S=10$ à 20); parmi les ions alcalino-terreux, le baryum donne les meilleurs résultats $(S \sim 100)$.

La solubilité des verres phosphates mixtes :

- diminue pour une même formule, quand le poids atomique du cation bivalent augmente;
- diminue quand la teneur du cation bivalent augmente;

- est faible $(S=10)$ pour les verres phosphates $\mathrm{Na}+\mathrm{Al}$

- les verres fabriqués suivant les brevets ( $§$ II.2 $e$ ) ont une très bonne stabilité, mais, comme déjà indiqué, la largeur de leur bande de fluorescence est aussi grande que celle des verres silicates;

- les verres germanates et les verres aluminates sont peu solubles, mais leurs spectres de fluorescence sont flous.

III.6. Effet laser. - Les verres phosphates décrits ci-dessus ont des caractéristiques lasers remarquables : ainsi, malgré la mauvaise homogénéité de nos fontes, due à la faible capacité de nos équipements, des barreaux élaborés à partir de ces fontes ont donné d'excellents résultats :

a) Fongtionnement LASer En impulsion. - Des barreaux cylindriques de $5,5 \mathrm{~cm}$ de long et de $3 \mathrm{~mm}$ de diamètre ont été découpés à partir d'une coulée de composition $\left(\mathrm{PO}_{3}\right)_{6} \mathrm{ZnLi}_{4}$, préparée au laboratoire comme indiqué $\S$ II.2. Les extrémités, après polissage pour réaliser une structure à miroirs sphériques confocaux, ont été recouvertes de miroirs d'argent dont l'un a une transparence de $2 \%$. Excités par une lampe à éclair, droite, PEK. XE1-2, le seuil de fonctionnement laser est de 2,5 joules.

A la suite de ces résultats, le laboratoire de la Société Sovirel, sous la direction de M. Meyer, a préparé deux fontes d'un litre de verre phosphate de la même composition. L'amélioration de l'homogénéité obtenue a entraîné un abaissement important du seuil de fonctionnement laser : des barreaux provenant de ces fontes (longueur $5 \mathrm{~cm}, \varnothing 0,3 \mathrm{~cm}$ ), munis de réflecteurs multidiélectriques, ont, à la température ambiante, des seuils de 1,02 joule. Cependant, l'homogénéité n'est pas encore idéale ainsi que l'indique le flou de la figure 8 qui représente les « modes » émis par un barreau tels que l'on peut les observer sur un conver-

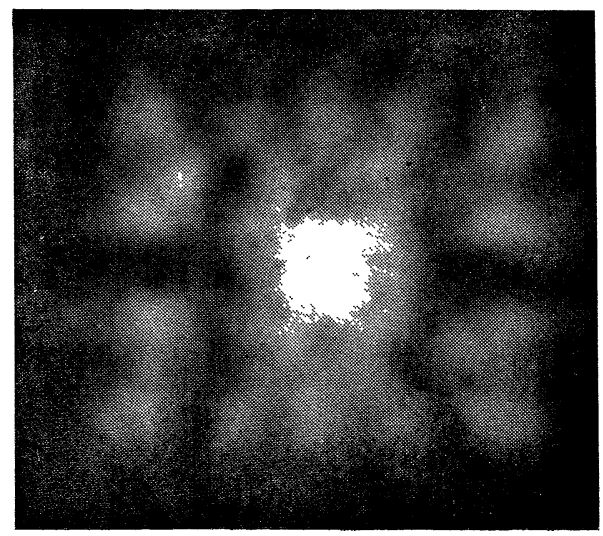

FIG. 8

Modes de l'émission d'un barreau de verre phosphate. 
tisseur d'image infrarouge. Avec des coulées encore plus homogènes nécessitant un volume plus élevé, le seuil laser serait certainement encore plus bas. A notre connaissance, ces seuils sont les plus bas que l'on ait obtenus avec des barreaux de verre laser. Par exemple, le seuil le plus bas, cité dans l'article de Maurer (1. c., fig. 8), est de 8 joules pour un verre silicate au $\mathrm{Ca}$ et $\mathrm{Na}$.

b) FonGtionNEMENT EN GONTINU A LA TEMPÉRATURE AMBIANTE. - L'effet laser quasi continu a été obtenu avec ces barreaux dans un appareillage décrit par Deutschbein et autres [16], déjà utilisé pour des cristaux de $\mathrm{CaWO}_{4}$. L'excitation est fournie par une lampe à arc de Xénon, d'une puissance nominale de $1 \mathrm{~kW}$, fonctionnant en alternatif $50 \mathrm{~Hz}$. En utilisant une alternance sur deux, une puissance électrique appliquée à la lampe de $720 \mathrm{~W}$ est suffisante pour

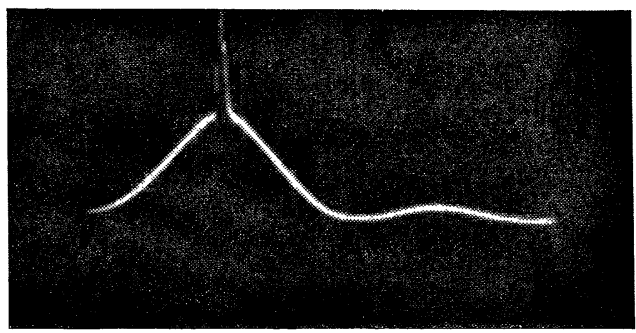

FIG. 9. - Variation de l'émission d'un barreau en fonction du temps.

obtenir l'effet laser; dans la figure 9, on aperçoit l'émission laser, au rythme de $50 \mathrm{~Hz}$. Avec les deux alternances le seuil des barreaux actuels est de $1100 \mathrm{~W}$.

G'est la première fois, à notre connaissance, que l'effet laser quasi continu, à la température ambiante, est obtenu avec des barreaux de verre de telles dimensions : $3 \mathrm{~mm}$ de diamètre ${ }^{\mathbf{1}}$ ). Nos barreaux supportent sans dommage des puissances de pompage de $1 \mathrm{~kW}$, mais des puissances supérieures entraînent une détérioration du barreau due à un échauffement excessif de celui-ci.

IV. Conclusion. - Cette recherche a prouvé que les propriétés des verres phosphates sont très différentes de celles des verres silicates et borates étudiées par de nombreux chercheurs. Ces différences concernent principalement : la finesse des spectres, la durée de vie du niveau laser initial et les probabilités de transition pour les transitions de pompage.

Ces verres présentent des performances lasers meilleures que celles des verres silicates et borates bien connues.

V. Remerciements. - Nous remercions la Société Sovirel et, en particulier, M. H. Meyer, directeur du laboratoire Sovirel (Le Vésinet), d'avoir préparé des fontes de verres phosphates, d'après nos formules, de bonne homogénéité optique.

Manuscrit reçu le 28 octobre 1966.

(1) Young a obtenu l'effet laser quasi continu avec des fibres de verre dont le diamètre utile était soit de $100 \mu$ [17], soit de $400 \mu[18]$, ces dernières ayant une durée de fonctionnement de $7 \mathrm{~ms}$. Ces résultats ne sont pas directement comparables aux nôtres, puisque, à puissance de pompage donnée, la différence de température entre l'axe et la surface du barreau, qui est la cause de sa détérioration, est proportionnelle au carré du rayon de celui-ci.

\section{BIBLIOGRAPHIE}

[1] SNitzer (E.), Phys. Rev. Letters, 1961, 7, 444.

[2] SNITZER (E.), Applied optics, 1966, 5, no 10, 14871499 .

[3] Maurei (R. D.), Proc. Symp. Opt. Masers, New York, 1963, 435.

[4] TOMASChEK (R.) et DEUTSChBEIN (O.), Glastechn. Berichte, 1938, vol. 16, 155.

[5] Trans. Faraday Soc., 1939, 35, 148.

[6] Deutschbein (O.) et Pautrat (Ch.), Onde électrique, avril 1966, vol. XLVI, no 469.

[7] VAN Wazer (J. R.), Phosphorus and its compounds, vol. 1. Chemistry, Interscience, New York, 1958

[8] GRIMM et HoOpERT : U.S. pat 1964-629, 1934.

[9] Schotr, Brevet français no 1302-063.

[10] Schawlow (A. L.), Solid State Journal, juin 1961, vol. $2, \mathrm{n}^{\circ} 6,3-11$.
[11] Yariv (A.) et GoRdon (J. P.), P. IEEE, janvier 1963, 4-29.

[12] Johnson (L. F.), J. Appl. Physics, avril 1963, 34, partie 1, 897-909

[13] Deutschbein (O.) et Auzei (F.), 3 e Congrès d'électronique quantique, Paris, février 1963.

[14] MoRey (W.), Properties of Glass, Reinhold Publishing Corporation, New York, 1938.

[15] Surugue (J.), Techniques générales du laboratoire de Physique, Éditions du C.N.R.S., 1952, vol. III, 431.

[16] Deutschbein (O.), Grimouilie (G.), Pautrat (Ch.) et Petit-Le Du (G.), Rev. Phys. App., juin 1966, 1, 128-132.

[17] Young (C. G.), Appl. Phys. Lett., 15 avril 1963, 2, no $8,151-152$.

[18] Young (C. G.), Appl. Optics, juin 1966, 5, no 6, 993-997. 